\title{
SULLA ORIGINE DEI MICROSISMI DEL MEDITERRANEO $\left(^{*}\right)$
}

\author{
M. GIORGI - E. RosinI
}

La presente nota ha lo scopo di illustrare brevemente i risultati di alcuni particolari studi condotti presso l'Istituto Nazionale di Geofisica sulla origine dei microsismi del Mediterraneo.

È superfluo precisare che con il nome di " microsismi » o " agitazione microsismica " intendiamo riferirci soltanto alla oscillazione del suolo avente carattere permanente, più o meno regolare, con periodi ed ampiezze variabili, che è in stretta relazione con perturbazioni meteorologiche agenti più o meno lontano dal luogo di osservazione.

I risultati di indagini particolareggiate per ciò che concerne $i$ microsismi in generale e il problema della loro origine si trovano riportati in alcune nostre precedenti pubblicazioni $\left({ }^{1,2,3}\right)$. In uno di questi nostri lavori $\left({ }^{3}\right)$ abbiamo esposto ed ampiamente documentato i risultati di uno studio sistematico da noi condotto per risolvere il problema fondamentale della individuazione della causa o delle cause che generano i microsismi, problema verso il quale si sono principalmente rivolti da molti decenni gli sforzi dei ricercatori. Fra questi è interessante ricordare un recente contributo di Caloi $\left({ }^{4}\right)$.

Nel citato lavoro abbiamo cominciato col fare una analisi critica degli abbondanti studi sui microsismi ed abbiamo rilevato come certi interessanti risultati di alcune ricerche teoriche e sperimentali, come quelle di Gherzi, Ramirez, Macelwane, Gutenberg, Lee, Don Leet, Ewing ed altri, hanno consentito di focalizzare sempre meglio il problema della correlazione tra attività microsismica e perturbazioni atmosferiche. Gli studi sui microsismi, tuttavia, si sono sempre limitati all'osservazione del comportamento dei diversi singoli elementi legati a dette perturbazioni in relazione con l'attività microsismica; così l'azione dei frangenti contro le coste, lo stato del mare, la pressione, il vento, $i$ centri depressionari, le perturbazioni frontali, ecc., hanno

(*) Comunicazione presentata alla IX Assemblea Generale dell'U.G.G.I. tenutasi a Bruxelles dal 19 al 31 agosto 1951. 
costituito elementi separati con i quali a molti studiosi non è stato difficile trovare una documentazione che avallasse le loro affermazioni sulle cause dei microsismi da loro presunte; era anzi impossibile non trovare correlazioni che non provassero le diverse ipotesi; detti elementi infatti sono in genere concomitanti e non si presenta facile una discriminazione fra essi. Gli elementi meteorologici singolarmente presi perdono ogni significato; la vicenda meteorologica è un risultato della loro composizione e della mutua azione che li fa interdipendenti;

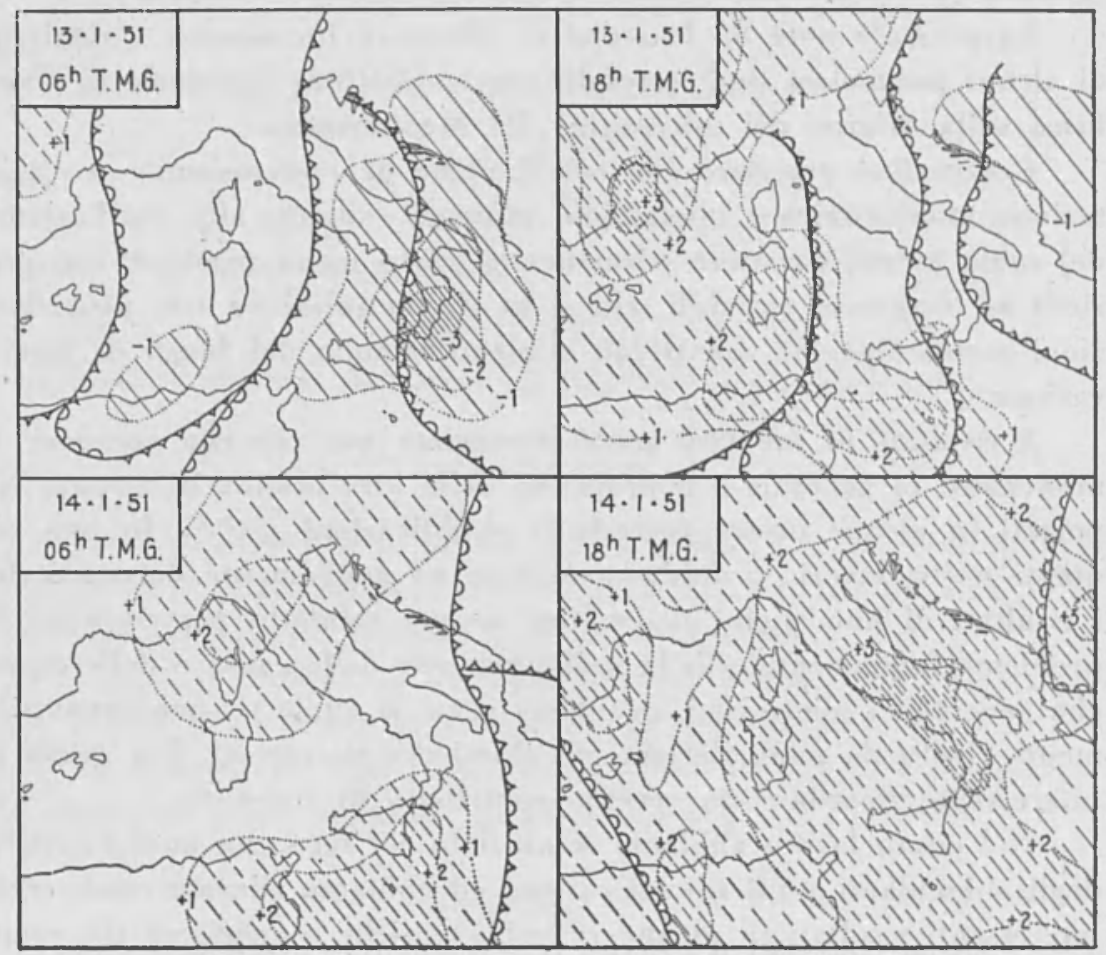

Fig. I

quindi gli elementi considerati separatamente non avrebbero mai potuto fornire una vera prova.

La sola via efficace da intraprendere per risolvere in maniera decisiva il problema della individuazione della causa dei microsismi era dunque quella di eseguire un esame minuzioso ed approfondito delle situazioni meteorologiche tenendo conto di tutti gli elementi che le determinano, confrontati con la contemporanea attività microsismica.

A tale scopo l'organizzazione della rete sismica nazionale dell'Istituto Nazionale di Geofisica e quella meteorologica del Servizio Me- 
teorologico dell'Aeronautica Italiana hanno fornito, nella maniera più ampia ed agevole, le prestazioni, il materiale e la documentazione necessaria.

Per le notizie concernenti i metodi ed i mezzi adoperati nella nostra ricerca rimandiamo alla nota già citata $\left({ }^{3}\right)$.

Lo studio che si presentava così laborioso, perché potesse condurci a risultati sicuri e precisi, è stato limitato ai microsismi provenienti dal settore per cui maggiore era la abbondanza dei dati meteorologici diretti o di elaborazione: cioè dal Mediterraneo; il criterio che abbiamo seguito e che ci è parso meglio resistente ad ogni critica non è stato quello di scegliere esempi singoli indiscutibilmente favorevoli ad una determinata tesi, ma di procedere allo studio parallelo dell'andamento dell'attività microsismica e della evoluzione contemporanea delle vicende meteorologiche con continuità per un lungo tempo di osservazione che oramai è di circa tre anni. In tal modo è stato possibile pervenire a risultati oggettivi derivanti da una ricerca sistematica e non conseguenza di una ipotesi aprioristica. Detti risultati possono così riassumersi :

a) l'ineguale intervallo di tempo che costantemente si osserva tra il passaggio di un centro depressionario sul mare, quando esiste, ed il sorgere di una attività microsismica sta a confermare che questa non dipende da esso;

b) non sussiste diretta dipendenza tra il passaggio di fronti a carattere freddo ed attività microsismica;

c) non esiste dipendenza alcuna dell'attività microsismica con fronti a carattere caldo;

d) infine non esiste alcuna correlazione costante tra forza del vento, direzione del vento, stato del mare da una parte ed attività microsismica dall'altra;

e) la quasi totalità dei casi esaminati ha invece indicato in modo assolutamente evidente la dipendenza diretta dell attività microsismica dalla presenza, estensione ed intensità dei nuclei di tendenza barometrica positiva in mare aperto; $i$ pochi restanti casi in cui questa evidenza non è parsa così certa ed esclusiva pur tuttavia non costituiscono prova contraria; è importante notare infatti che anche in questi casi tale elemento è sempre esistito anche se non presentava un'apparente netta prevalenza rispetto agli altri elementi. In altre parole detto elemento è stato sempre presente e solo in qualche caso le osservazioni non hanno permesso una determinazione causale più precisa ed univoca. 
Il risultato fondamentale a cui siamo pervenuti, è pertanto il seguente: $i$ microsismi di origine mediterranea vengono generati nelle zone in mare aperto interessate da nuclei di tendenza barometrica positiva, stazionari o in movimento.

Una volta individuata la causa specifica abbiamo potuto inoltre provare in maniera molto più esauriente e diretta di quanto ai vari ricercatori fino ad ora non è era stato possibile, che il periodo iniziale dell'agitazione microsismica viene determinato dal luogo ove ha

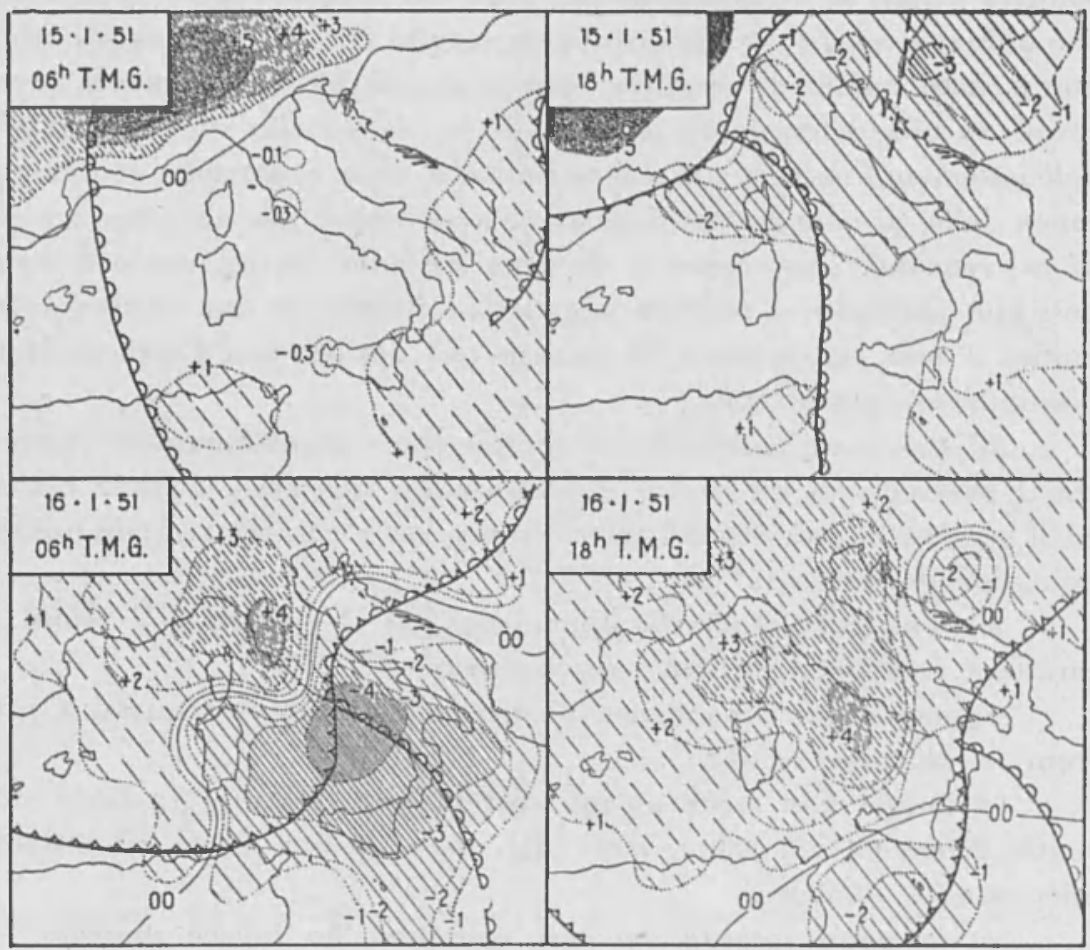

Fig. 2

origine l'agitazione stessa e rappresenta un elemento fondamentale caratteristico legato alle modalità della generazione dell'oscillazione microsismica, alla intensità del nucleo nonché alla profondità delle acque marine e delle caratteristiche sismo-elastiche e di struttura del fondo solido del mare e dello zoccolo continentale. Detto periodo subisce poi variazioni dello stesso senso delle variazioni sia della distanza sia della intensità della agitazione; ed inoltre una agitazione sorta in concomitanza con una perturbazione atmosferica, pur variando 
successivamente di ampiezza e di periodo a seconda delle variazioni della energia e delle posizioni dei nuclei di tendenza positiva mantiene sempre un suo carattere morfologico specifico.

Riportiamo qui, a titolo di esempio, e per ovvi motivi limitata, una documentazione parziale dei nostri risultati che si riferisce ad alcune tempeste microsismiche verificatesi nel Mediterraneo centrale dal 13 al 18 gennaio 1951.

Tempeste microsismiche del 13-18 gennaio 1951. - Nel periodo 13-18 gennaio 1951, in occasione del passaggio di numerose perturba-

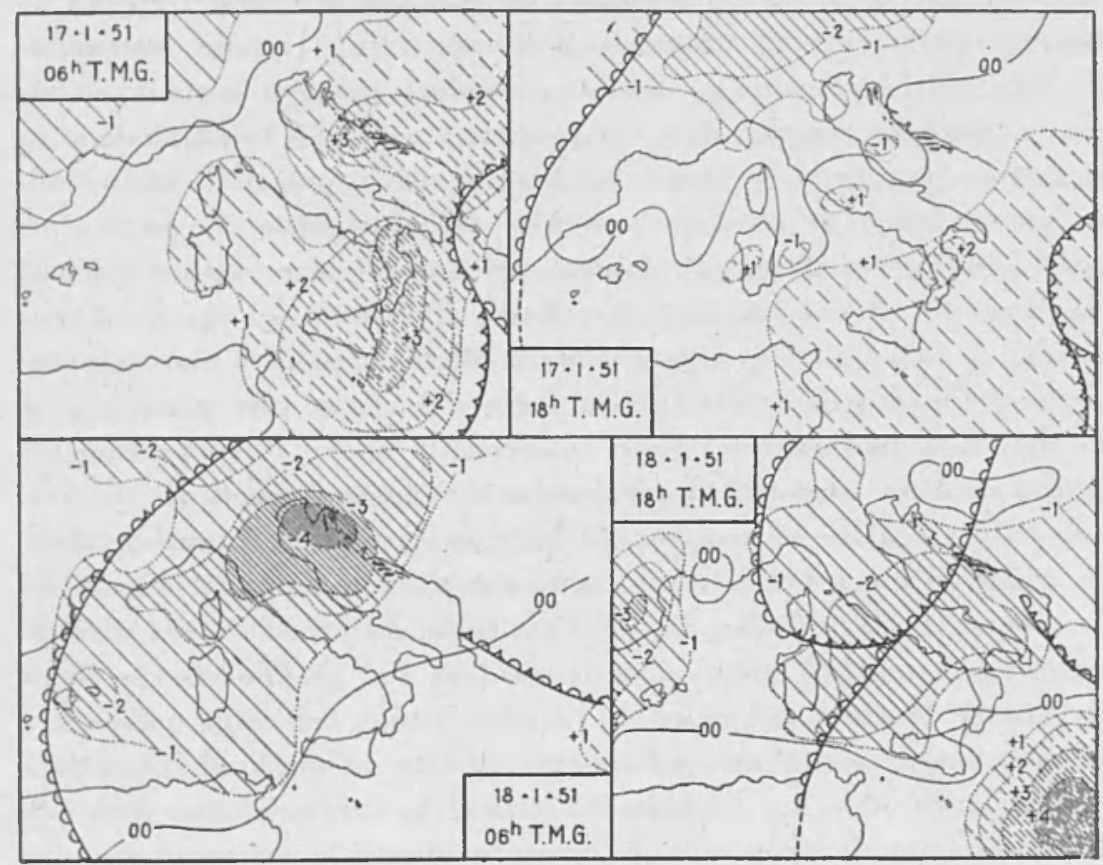

Fig. 3

zioni e fronti attraverso il Mediterraneo, l'agitazione microsismica registrata a Roma I.N.G. ha presentato un andamendo che si presta bene per l'individuazione delle cause che la generano.

A cominciare dalle ore 06 T.M.G. del 13 gennaio, si constata la presenza di una serie di occlusioni in marcia verso levante precedute da nuclei negativi delle tendenze barometriche di cui uno intenso che ha già attraversato il Tirreno meridionale. Sia l'occlusione centrale che il nucleo negativo mostrano di non essere attivi nei riguardi della agita- 
zione microsismica; a tal riguardo occorre tener presente che l'occlusione non è seguita da un apprezzabile nucleo di tendenze positive. Le perturbazioni posseggono una velocità notevole, specie la più occidentale, che 12 ore dopo si trova già in pieno Tirreno seguita da un nucleo positivo vasto $\mathrm{ma}$ in realtà piuttosto debole, come si riconosce considerando che alle ore 18 T.M.G. tutto il campo barico è in aumento. Si affaccia però sul golfo del Leone un nucleo positivo più netto che ben presto si inoltra nel Mediterraneo settentrionale; con esso si inizia l'agitazione microsismica. Da questo momento lo spostamento del nucleo e l'andamento della agitazione mostrano un completo parallelismo: il massimo della agitazione si verifica alle ore 08 del giorno 14 contemporaneamente al passaggio del nucleo sul Tirreno settentrionale, quando l'occlusione a carattere freddo è già ben lontana ed attenuata. Successivamente, durante la giornata del 14 l'agitazione è in diminuzione piuttosto lenta ed il nucleo positivo, piegando verso SudEst, scorre lungo il versante tirrenico dell'appennino. E particolarmente significativo il comportamento dei dati di osservazione rilevati a Livorno nei giorni considerati, relativi allo stato del mare (di cui mancano i valori notturni perché dalla stazione non si può valutare il mare nelle ore di oscurità), alla forza del vento, alla pressione ed alla tendenza barometrica. Sono interessanti anche $i$ comportamenti dei dati di altre stazioni, ed a tal uopo si riportano quelli di tre Stazioni dislocate a larghe maglie: Alghero nęlla Sardegna nord-occidentale, Cozzo Spadaro nell'estrema punta meridionale della Sicilia e l'Isola di Ponza al larơo di Napoli, però fra tutte, Livorno è la più direttamente interessata all'intera vicenda. Si trae dal grafico che la forza del vento e lo stato del mare di Livorno, come del resto nelle altre Stazioni, hanno avuto un andamento opposto a quello dell'intensità dell'agitazione; solo per Alghero e Ponza si ha una analogia, però con uno sfasamento di molte ore. E invece evidente la concomitanza fra il massimo della tendenza barometrica ed il massimo dell'agitazione. Dunque in questa prima parte del periodo preso in esame si è avuta una tempesta microsismica completa nelle sue fasi e visibilmente non vincolata al passaggio della perturbazione frontale a carattere freddo né al vento ed allo stato del mare, ma strettamente legata al comportamento di un nucleo di tendenze positive assai ben caratterizzato. Non meno probante ̀̀ la connessione fra l'evoluzione della situazione meteorologica e la tempesta microsismica che, appena placatasi la precedente, si è prodotta con violenza assai maggiore nei giorni successivi.

Durante la giornata del 15 i sismograf hanno registrato una assai 
debole oscillazione della crosta terrestre mentre un fronte caldo spazzava il Mediterraneo occidentale ed il Tirreno. Ma dietro il corrispondente fronte freddo si è delineato un forte nucleo di tendenze positive proveniente dall'Atlantico ed in cammino attraverso la Francia con direzione verso SE. Nella serata del giorno 15 il punto ciclonico della perturbazione è giunto sulla Liguria ed il fronte freddo ha fatto irruzione nel Mediterraneo a cominciare dal Golfo del Leone. La velocità di spostamento del fronte freddo è tale che la mattina del giorno 16 lo si trova già sul Tirreno centro-meridionale e 12 ore dopo sullo Jonio: cioè dalle ore 18 del giorno 16 in poi il fronte freddo non interessa più il Tirreno ed il canale di Sicilia e pertanto da tale ora, fino al giorno 18 , la zona di produzione dell'agitazione microsismica in esame è sgombra da perturbazioni frontali. Se l'agitazione microsismica fosse collegata direttamente al passaggio del fronte freddo, la sua massima intensità si sarelbbe registrata fra le ore 18 del giorno 15 e le ore 18 del giorno 16 , attesa anche l'energia notevole del fronte. L'agitazione microsismica invece, debolissima fino alle prime ore del giorno 16 , inizia in tali ore la sua intensificazione e raggiunge una vistosissima ampiezza nella serata del 16 mantenendosi poi immutata per molte ore e diminuendo gradualmente nella seconda metà del giorno 17.

Neppure l'andamento dei venti e dello stato del mare nelle località prescelte come più significative mostra una definita concordanza con la tempesta microsismica: a Livorno la forza del vento rimane tenue in tutto il periodo e lo stato del mare diminuisce di intensità dal giorno 16 al 17. A Ponza, a Cozzo Spadaro e ad Alghero si hanno venti forti e fortissimi a cominciare dal giorno 15 e per tutta la giornata del 16, ma lo sfasamento con l'andamento della agitazione microsismica è evidente e notevole: quasi 12 ore per Alghero, circa 6 ore per Cozzo Spadaro e Ponza. Questo andamento dei venti, come anche l'andamento dello stato del mare, è in piena concordanza con le ore di passaggio del fronte freddo nelle rispettive località. Esclusi dunque tutti questi elementi meteorologici come cause dirette del movimento del suolo, si riconosce subito che, al contrario, l'ampiezza del movimento si accorda perfettamente alle evoluzioni di un netto nucleo positivo che segue il fronte freddo ad una distanza di $300-400 \mathrm{~km}$. Dalle cartine si vede come tale nucleo, ancora tutto nell'entroterra francese alle ore $18 \mathrm{del}$ giorno 15 , vada poi ad interessare l'Italia nord-occidentale e successivamente entri decisamente nel Tirreno centrale per dirigersi infine con il suo centro verso lo stretto di 


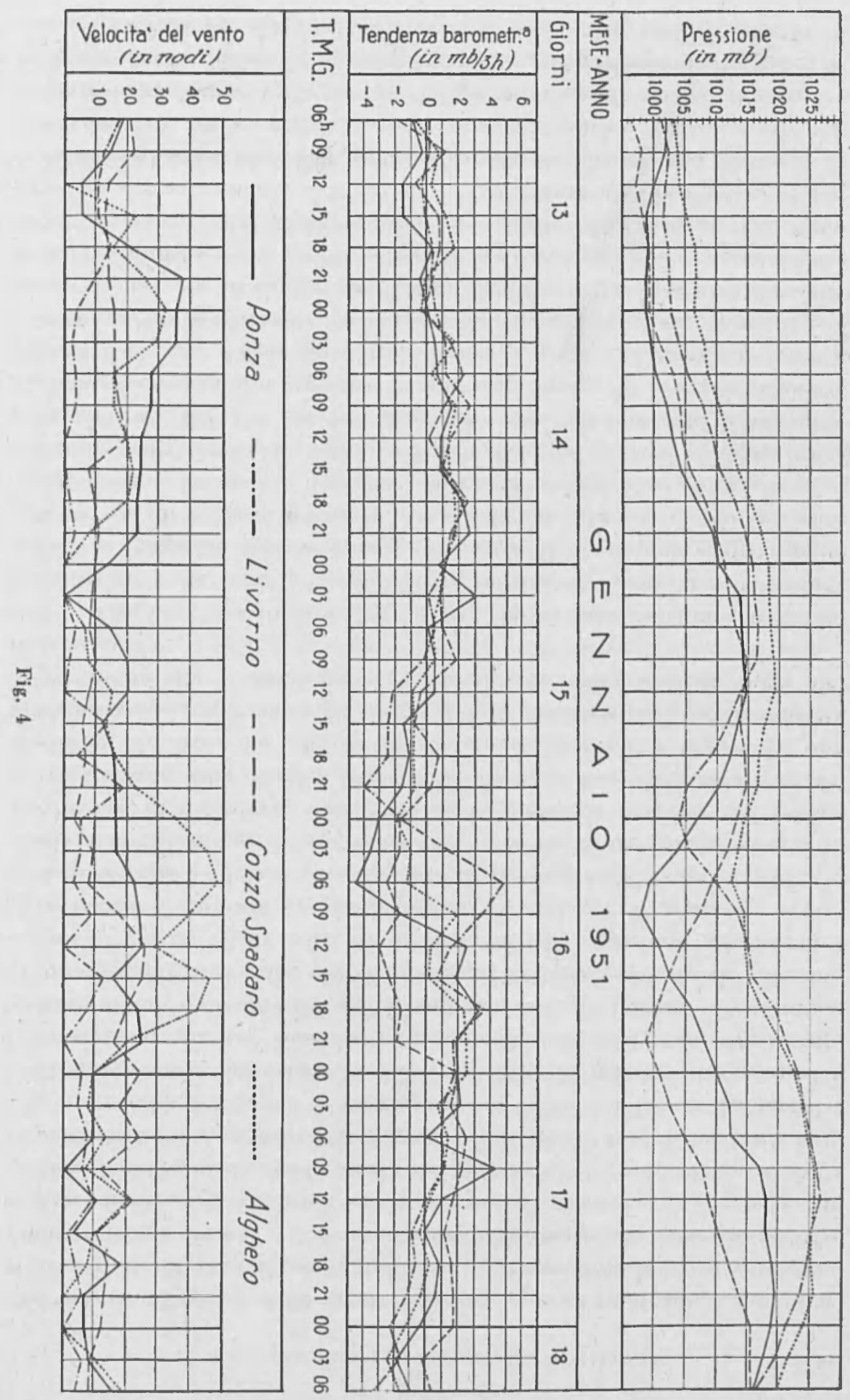




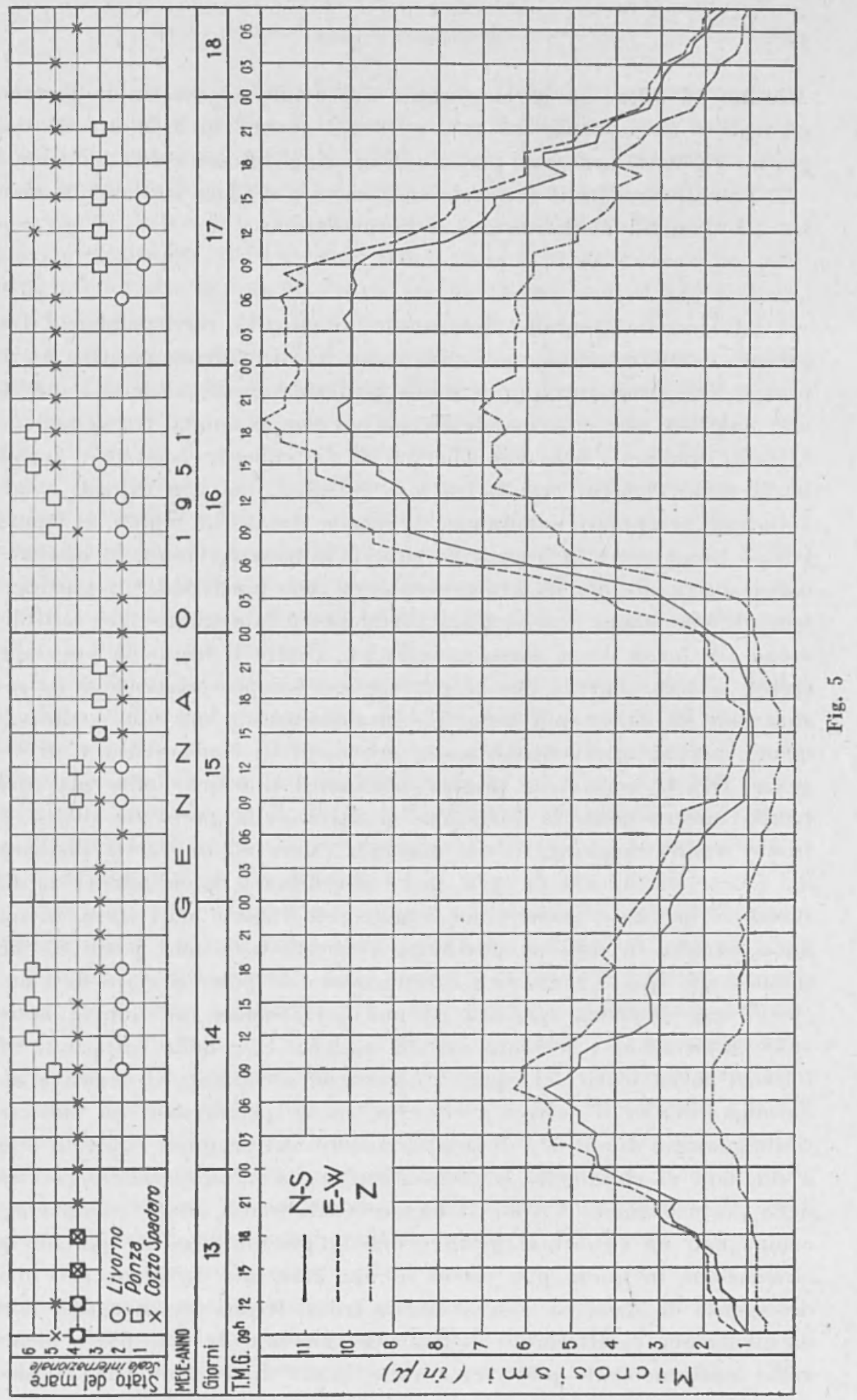


Messina ed oltre. La parte centrale del nucleo attraversa il Tirreno centrale e meridionale fra le ore 18 del giorno 16 e le ore 06 del giorno 17 , esattamente nel periodo di massima intensità dell'agitazione.

Successivamente la tempesta microsismica si placa lentamente mentre gli elementi meteorologici si normalizzano.

La ricerche condotte dimostrano dunque la corrispondenza fra agitazione microsismica e presenza di nuclei di tendenze positive sopra i mari. Tale risultato rappresenta un perfezionamento rispetto a quello clie stabilisce una corrispondenza, già da tempo notata, fra agitazione e fronti freddi. E ben noto che nuclei di tendenze positive e fronti freddi sono elementi spesso fra loro collegati, ma ben distinti e talvolta non accoppiati, comunque, di regola, sfasati nel tempo. Il fronte freddo rappresenta la linea sulla superficie terrestre lungo la quale le masse d'aria che per la loro provenienza sono più fredde (o più esattamente che hanno temperatura pseudopotenziale minore) si sostituiscono alle meno dense masse preesistenti. Dietro il fronte la pressione cresce, sostanzialmente perché per spessori sempre crescenti si ha sostituzione di masse più pesanti: sostanzialmente, ma non esclusivamente, perché intervengono anche fenomeni di convergenza e divergenza. Ora la quotidiana pratica dell'analisi sinottica delle carte del tempo mostra come la variazione positiva della pressione dietro il fronte freddo raggiunga il suo massimo valore ad una certa distanza dal fronte stesso, non di rado anche a centinaia di chilometri di distanza. E ben noto anche come $i$ meteorologi siano stati tratti da un buon numero di fatti ad attribuire a queste variazioni positive, che si mostrano spesso tanto ben caratterizzate da poter parlare di ( nuclei ", una esistenza specifica ed una permanenza individuale notevole (si ricordino i cosidetti metodi isallobarici e delle variazioni ed i lavori anche teorici al riguardo). I nuclei accoppiati, il negativo all'avanguardia ed il positvo a distanza, spesso percorrono con indiscutibile analogia di entità e di comportamento dei cammini notevoli, fino a migliaia di chilometri, seguendo con le loro fasi parallele la vita della perturbazione. A volte si hanno fronti freddi anche energici accompagnati da variazioni positive deboli, presumibilmente perché la circolazione in quota può essere in una fase tale da provocare una divergenza di masse; a volte si hanno fronti freddi accompagnati non da nuclei ben caratterizzati di tendenze, ma solo da aree più o meno vaste uniformemente positive; a volte infine dietro fronti freddi de- 
boli, o al limite anche inesistenti al suolo, si vedono comparire cospicui nuclei positivi, indice dell'esistenza del fronte in quota o di una forte convergenza in quota. In tutti questi casi di discordanza fra fronti freddi e nuclei positivi, e tutte le volte che questi due elementi siano molto distanziati geograficamente, si può constatare che l'agitazione microsismica va d'accordo con $\mathrm{i}$ nuclei in maniera assai più soddisfacente che non con i fronti freddi. Questo è il risultato delle ricerche sistematiche condotte; la documentazione completa, per ovvî̀ motivi, non può essere prodotta in questa sede, ma sia permesso sottolineare come ad una affermazione del genere possa giungersi, come abljiamo già detto, solo attraverso un esame sistematico che non si limiti alla scelta di alcuni casi interessanti ma albbracci nella sua interezz u un periodo sufficientemente lungo, e che non consista nell'andare a vedere quale sia la situazione meteorologica quando i sismografi accusano una agitazione imponente, ma viceversa si basi sulla considerazione parallela e completa del tempo nel suo svolgimento ed in tutti $i$ suoi elementi al suolo ed in quota e dell'agitazione microsismica in tutti $i$ suoi mutevoli aspetti.

Naturalmente l'agitazione microsismica, così come i nuclei positivi delle tendenze barometriche, possono essere collegati ad una occlusione anziché ad un fronte freddo; ciò sembra essere spesso il caso delle agitazioni di origine atlantica specie se ad alte latitudini, dove spesso la perturbazione è già in stato di ben sviluppata occlusione. Anche questa circostanza, certo notata anche da altri, ha importanza per le considerazioni che seguono.

Proviamo ora a domandarci : quale è l'agente specifico che genera i microsismi e. quale il meccanismo della sua azione? A noi sembra che i sopradescritti risultati della nostra indagine suggeriscano delle ipotesi abbastanza ragionevoli. Il fatto che l'agitazione microsismica sia concomitante non ai fronti freddi ma ai nuclei, che generalmente li seguono, ci fa ritenere che l'azione non sia di natura superficiale ma parte da una quota più o meno elevata. In altre parole non è l'azione diretta della massa fredda sulla superficie del mare sulla quale avanza, anzi proprio sulla linea dell'avanzata; non è di conseguenza neppure da pensare ad un eventuale effetto dinamico dovuto alla rotazione del vento che accompagna il passaggio del fronte.

Forse qualche volta questo effetto può aversi, ovviamente, e sembra risolversi in una agitazione contorta ed irregolare di piccola ampiezza e senza periodo definito: ma non si tratta che di casi scarsamente vistosi e locali. Invece acquista verosimiglianza l'ipotesi che 


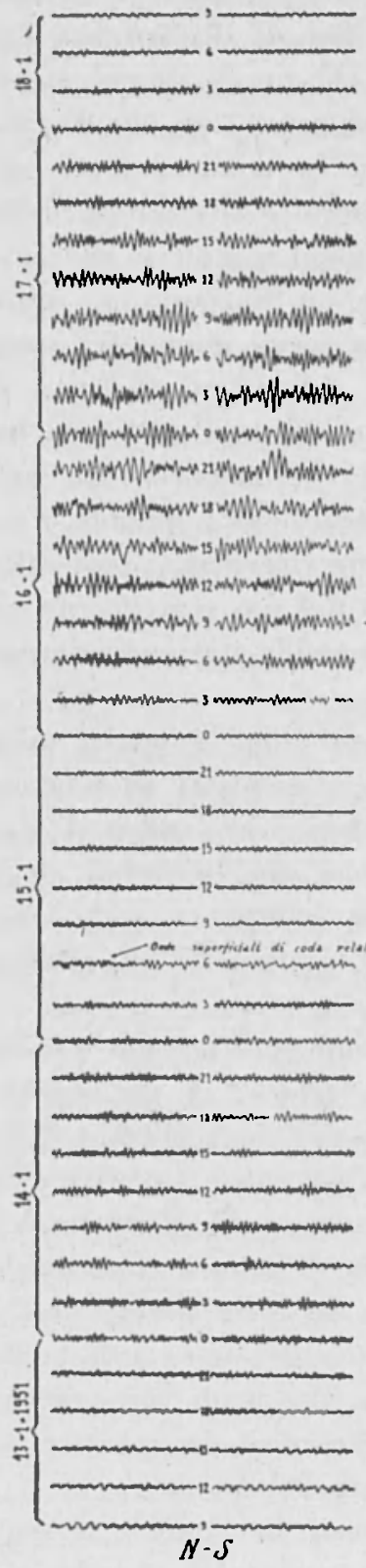

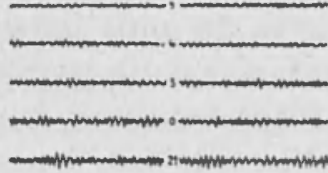

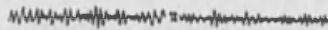

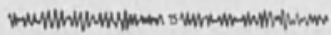

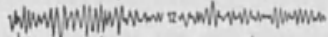

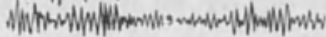

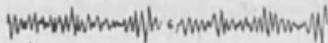

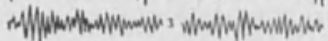

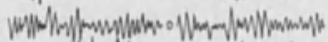

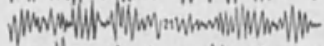

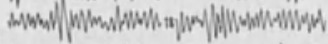

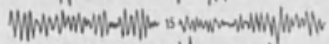

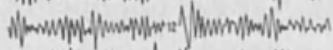

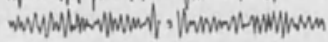

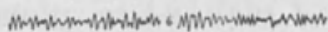

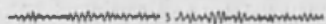

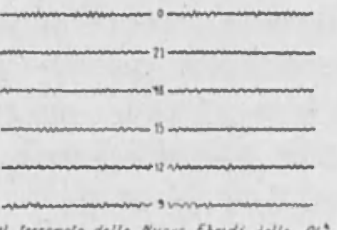

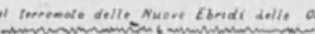

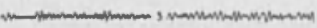

Antupum

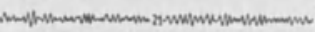

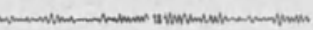

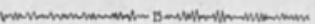

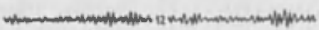

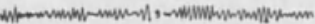
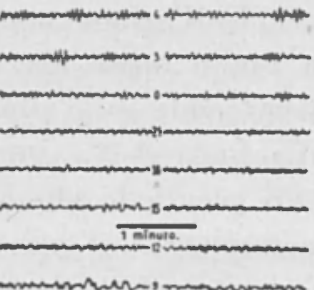

$E \cdot W$

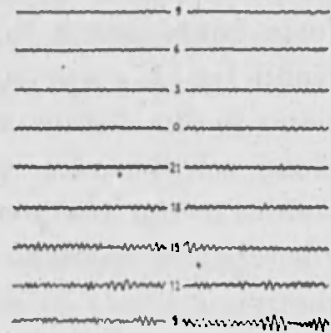

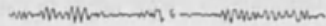

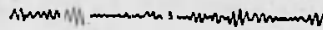

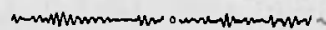

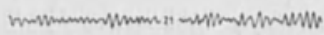

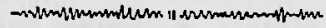

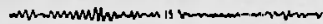

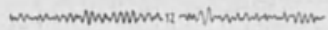

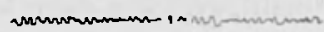

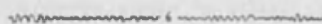
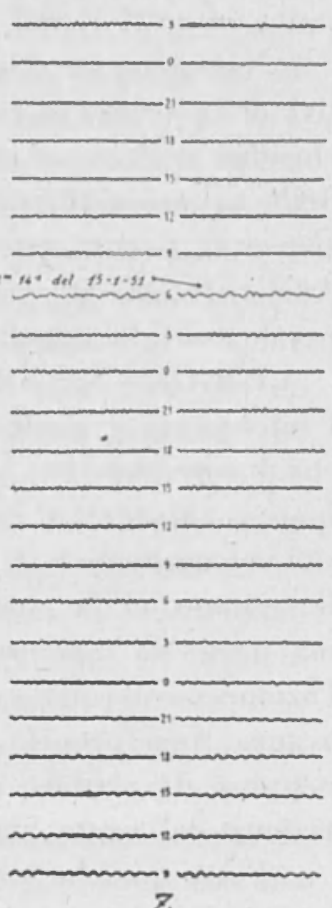

Fig. 6 - Andamento dei microsismi registrati a Roma durante le tempeste dal 13 al 18 gennaio 1951 
l'energia venga trasmessa alla superficie marina dall'alta quota attraverso lo strato atmosferico compreso. E hen nota la principale differenza morfologica fra le superficie frontali calde, regolari, prive di turbolenza, accompagnate da nubi stratificate e venti costanti, e le superfici frontali fredde turbolente, con moti convettivi attivissimi, con nubi cumuliformi e cumolonembi e venti tipicamente a raffiche.

Che l'agitazione segua a distanza il passaggio del fronte freddo può significare che effetti notevoli ai fini della produzione dei microsismi si possono avere solo quando la massa fredda turbolenta abbia raggiunto uno spessore considerevole. Sotto questo aspetto le nostre ricerche, estese all'analisi dei sondaggi termodinamici indicherebhero un effetto massimo per spessori da 1 a $3 \mathrm{~km}$. Ma che l'agitazione sia concomitante con la massima velocità di variazione della pressione barometrica, indicata dalla zona centrale del nucleo di tendenze positive, può indicare qualcosa di ancor più significativo: la zona di tendenze massime si ha quando le superfici frontali raggiungono, nella libera atmosfera, una quota tale che l'effetto della sostituzione delle masse fredde alle calde risulti massimo (a quote superiori si attenua evidentemente perché la densità di ambo le masse diviene troppo tenue). Ora è ragionevole supporre, benché su questo punto la meteorologia non sia ancora in grado di dare notizie esaurienti, che la zona in quota, dove la sostituzione di masse ha effetti barici più cospicui, cioè in corrispondenza dei nuclei, sia anche la zona dove le ondulazioni della superficie di separazione sono più ampie e potrebbero esercitare un effetto di " pumping" analogo a quello ipotizzato da P. Ghezzi nel caso di cicloni tropicali. D'altra parte a quelle quote può essere scomparso l'effetto perturbante della superficie terrestre e le ondulazioni possono risultare più regolari. Insomma la sostituzione di lenti d'aria alternativamente calda e fredda produrrebbe impulsi di pressione più o meno regolari alla superficie del mare del tipo di onde sonore; il mare poi a seconda della sua profondità e larghezza risuona per diversi periodi che trasmette al fondo.

Si può anche, forse più verosimilmente, pensare che detti impulsi di pressione possono generarsi dove la tendenza harometrica è massima perché ivi si ha pure un massimo di agitazione e turbolenza dell'atmosfera.

Roma - Istituto Nazionale di Geofisica - Agosto 1951. 


\section{RIASSUNTO}

La nota contiene $i$ risultati di indagini particolareggiate condotte presso l'Istituto Nazionale di Geofisica con la collaborazione del Servizio Meteorologico dell'Aeronautica sul problema dell'origine dei microsismi. Lo studio concerne fondamentalmente $i$ microsismi aventi origine nel Mediterraneo perché questo è il settore per cui maggiore era la abbondanza dei dati meteorologici e di più diretto controllo. L'osservazione continua di oltre tre anni conferma oramai il risultato più importante: $i$ microsismi di origine mediterranea non sono in diretta correlazione con $i$ fronti bensi vengono generati nelle zone, in mare aperto, interessate da nuclei di tendenza barometrica positiva.

Si riporta, a titolo di esempio, la documentazione relativa ad alcune tempeste microsismiche. Si dà poi una interpretazione delle modalità di generazione dei microsismi stessi e della trasmissione della energia dall'atmosfera, attraverso le masse marine, al fondo solido dell'Oceano.

Ricerche estese all'analisi dei sondaggi termodinamici stanno ad indicare che l'effetto massimo si ha quando lo spessore della massa fredda turbolenta ̀̀ fra 1 e $3 \mathrm{~km}$.

\section{BIBLIOGRAFIA}

(1) Rosin E.: L'agitazione microsismica e le perturbazioni atmosferiche. Rivista di Meteorologia Aeronautica, A. VIII, n. 4, 30-40 (1948).

(2) Giongi M.: Su alcuni aspetti caratteristici dei microsismi a Roma in relazione con fattori meteorologici. Annali di Geofisica v. 2, 24-39, n. 1 (1949).

(3) Giong M. - E. Rosiñ : Sulla natura delle cause che generano $i$ microsismi. Annali di Geofisica, v. 3, n. 2, 173-213 (1950).

(t) Calor P.: Due caratteristici tipi di microsismi. Annali di Geofisica, v. 3, n. 3, 303-314 (1950). 